\title{
Mechanism Underlying Specificity of Proteins Targeting Inorganic Materials
}

(Supporting Information)

Tomohiro Hayashi ${ }^{1,2}$, Ken-Ichi Sano ${ }^{3}$, Kiyotaka Shiba ${ }^{3}$, Yoshikazu Kumashiro ${ }^{1,2}$, Kenji Iwahori $^{4}$, Ichiro Yamashita ${ }^{4,5}$,and Masahiko Hara ${ }^{1,2, *}$

${ }^{1}$ Department of Electronic Chemistry, Interdisciplinary Graduate School of Science and Engineering, Tokyo Institute of Technology,

${ }^{2}$ Local Spatio-Temporal Functions Laboratory, Frontier Research System, RIKEN (The Institute of Physical and Chemical Research)

${ }^{3}$ Department of Protein Engineering, Cancer Institute, Japanese Foundation for Cancer Research, and CREST, JST

Graduate School of Materials Science, Nara Institute of Science and Technology, and CREST, JST

${ }^{4}$ Graduate School of Materials Science, Nara Institute of Science and Technology, and CREST, JST ${ }^{5}$ Advanced Technology Research Laboratories, Matsushita Electric Industrial Co., Ltd.

*corresponding author

\section{Sample preparation}

$\mathrm{Au}(111)$ substrates were prepared by evaporating gold onto freshly cleaved mica substrates. Prior to use, we baked the gold substrate at $400{ }^{\circ} \mathrm{C}$ for 4 hours. SAMs of ODT and MUOH were formed by immersing gold substrates in the ethanol solution containing the corresponding molecules at a concentration of $1 \mathrm{mM}$ for 24 hours. The $\mathrm{Si}$ substrates were cleaned by ultrasonication in an acetone bath for $5 \mathrm{~min}$ and then in ethanol for $5 \mathrm{~min}$, immediately after which they were rinsed thoroughly with pure water and air-dried under flowing nitrogen. They were then further cleaned by UV-ozone exposure (UV-3000, SAMCO, Tokyo, Japan) for $15 \mathrm{~min}$ at room temperature. The Ti substrates were rinsed with ethanol and then cleaned by UV-ozone exposure for $15 \mathrm{~min}$. HOPG substrates were peeled with an adhesive tape prior to each experiment. Static water contact angles were measured with contact angle meter (DSA 10 Mk2, Krüss, Hamburg, Germany).

In the procedure, $1 \mathrm{mg}$ PBLH was solubilized in $0.68 \mu \mathrm{l}$ chloroform containing dichloroacetic acid $(0.54 \mathrm{mg} / \mathrm{mL})$. The desired amount $(10 \mu \mathrm{l})$ of PBLH was 
then spread over the recombinant ferritin, $\Delta 1$-LF or minT1-LF solution $(1 \mathrm{mg} / \mathrm{mL}$, Tris- $\mathrm{HCl}$ buffer at $\mathrm{pH} 8.0)$ in a Langmuir trough $(20 \times 45 \times 2 \mathrm{~mm})$ and allowed for condensed ferritin monolayer to adsorb onto the PBLH film for 3 hours at room temperature. The obtained hetero-bilayer of ferritin and PBLH was horizontally transferred onto the AFM tip, and the tip was then immediately stored in deionized water. To check the density of ferritins immobilized on the AFM tip, we performed an AFM observation of the hetero-bilayer of ferritin and PBLH on the V-shaped cantilever surface. A close-packed monolayer of ferritin, $\Delta 1$-LF or minT1-LF was always imaged under air. We therefore concluded that the AFM tip is fully covered with the monolayer of ferritin molecules. ${ }^{1}$

\section{Stability of the hetero-bilayer of PBLH and ferritin}

We also checked the stability of the bilayer during the AFM measurements very carefully using the variation of adhesion force between the tip and substrate. In this work, we carefully selected and employed AFM tips showing similar adhesion forces against HOPG $(1.5 \mathrm{nN})$ to avoid the effect of the differences in tip geometry. The selected tip fully covered with $\Delta 1$-LF or minT1-LF, showed the adhesion forces against Si substrates about 0.5 and $2.5 \mathrm{nN}$, respectively, at the same loading rate. After many approach and retract cycles, the adhesion force between the tip and Si substrate sometimes showed a sudden rise to a much higher value (above $4 \mathrm{nN}$ ). This is explained by the exfoliation of ferritin molecules from PBLH film, because the tip covered with PBLH showed similar adhesion force and a bare $\mathrm{Si}_{3} \mathrm{~N}_{4}$ tip exhibited almost no adhesion force. In most cases, the hetero-bilayers were stable for more than 100 cycles of approaches and retractions. With these findings taken together, we concluded that the ferritin is rigidly immobilized on the AFM tip. ${ }^{2}$

\section{Effect of solution conditions on the adhesion force}

We checked the effect of salt and $\mathrm{pH}$ value. Figure 1 and 2 show the adhesion force measured in Tris- $\mathrm{HCl}$ buffer $(\mathrm{pH} 8.0)$ with $\mathrm{NaCl}(150 \mathrm{mM})$ and in this buffer containing TWEEN20 $(0.5 \mathrm{wt} \%)$, respectively. We found there is little effect of the condition of the solution on the adhesion force, indicating that ions in solution do not play an important role in the adhesion event. 


\section{References \& Comments}

(1) Hayashi, T.; Hara, M. Jpn. J. Apl. Phys. 2005, 44, (7B), 5374.

(2) In the case of TWEEN20 added to the solution, we checked the immobilization of ferritin on the tip by going back to pure water and clean substrates, and examined the adhesion force. In most of the case, the adhesion force represented same value as before, indicating that the surfactant molecules are easily removed from the surface of ferritin molecules. 


\section{Figure Captions}

Figure 1s the adhesion forces measured in Tris- $\mathrm{HCl}$ buffer $(\mathrm{pH} 8.0,50 \mathrm{mM})$ with $\mathrm{NaCl}$ (150 mM).

Figure $2 \mathrm{~s}$ the adhesion forces measured in Tris- $\mathrm{HCl}$ buffer $(\mathrm{pH} 8.0,50 \mathrm{mM})$ with $\mathrm{NaCl}$ $(150 \mathrm{mM})$ containing TWEEN20 at a concentration of 0.5 weight percent 


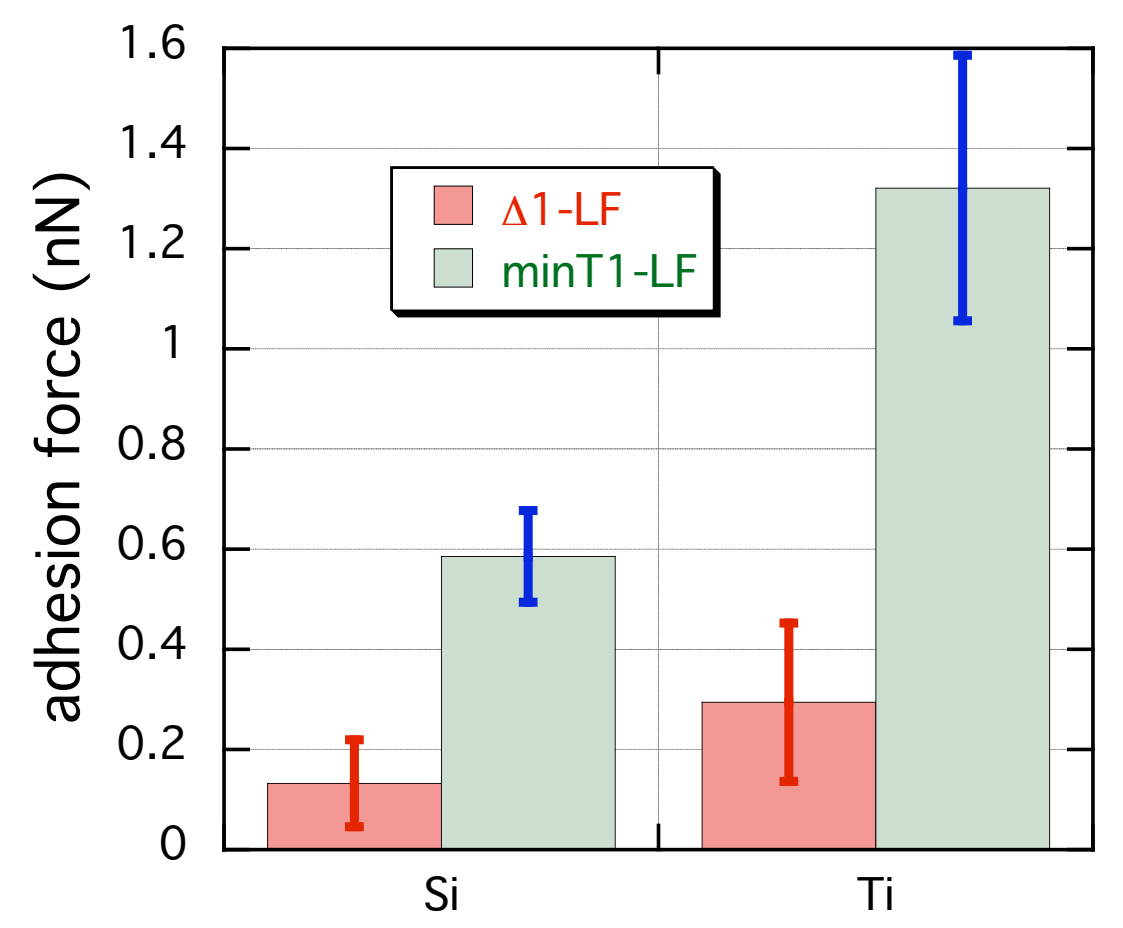

Figure 1s

Hayashi et al. 


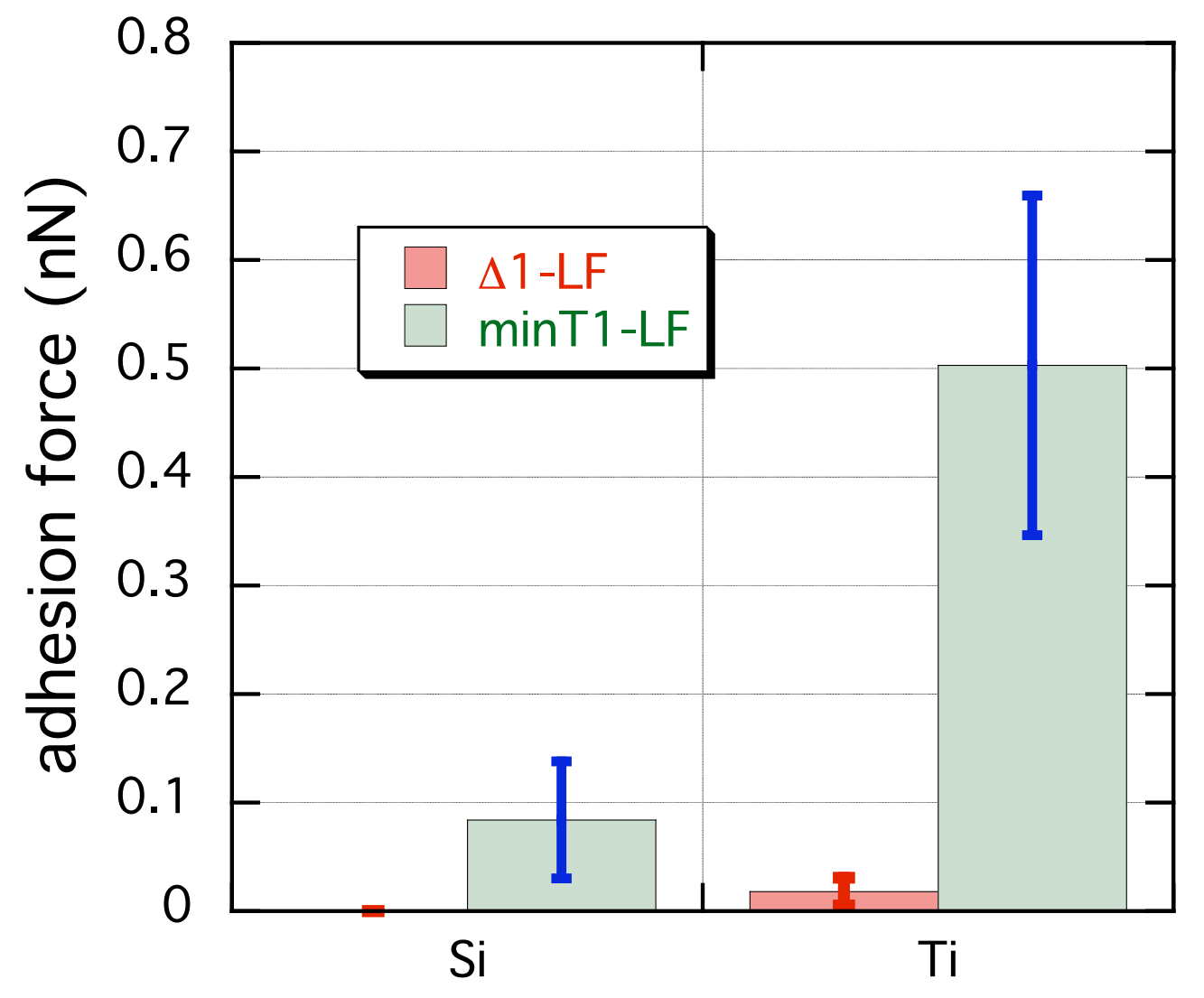

Figure 2s 